\title{
A Novel Emergency Healthcare System for Elderly Community in Outdoor Environment
}

\author{
Hui-Ru Cao iD and Choujun Zhan \\ Nanfang College of Sun Yat-sen University, Guangzhou 510970, China \\ Correspondence should be addressed to Hui-Ru Cao; xiaocao0924@163.com
}

Received 15 January 2018; Revised 20 May 2018; Accepted 8 July 2018; Published 22 July 2018

Academic Editor: Heng Qi

Copyright (C) 2018 Hui-Ru Cao and Choujun Zhan. This is an open access article distributed under the Creative Commons Attribution License, which permits unrestricted use, distribution, and reproduction in any medium, provided the original work is properly cited.

\begin{abstract}
By exploiting the advanced information and communication technologies, the current community healthcare systems provide digital healthcare services. However, the current healthcare framework for senior citizen in outdoor environment faces new challenges. The traditional healthcare systems are not efficient, and they do not comprise user-friendly devices and interfaces suitable for the elderly in outdoor environment. Hence, in this work, we develop an outdoor healthcare system for community senior citizens based on unmanned aerial vehicle (UAV) and Internet of things (IoT). Our system includes physical devices, wireless and wired networks, cloud and data center, and smart terminals. Further, the analysis of the proposed healthcare architecture is presented from the perspective of different layers, and an algorithm on UAV for creating high-speed communication channel and delivering medicine is provided. In addition, the healthcare UAV, related devices, and friendly APPs are designed. The proposed framework is evaluated by comparison with the current healthcare architecture. The emulating and experimental results show that our proposed system can provide a high-quality wireless communication link even at large communication distances, and in real testbed our proposal can reduce the response time by about $20 \%$ in comparison with the current methods.
\end{abstract}

\section{Introduction}

With the increase of life expectancy in many countries, the continuously growing aging trends bring a dramatic increase in the elderly population [1]. Specifically, most of the developing countries are facing a serious aging problem, and more and more senior citizens suffer from the acute conditions such as blood pressure, heart disease, and fall-related injuries [2]. On the other hand, in most developing countries, most of the younger generation move to big cities for work, and this leads to a large number of old people living alone without family. This has become a common phenomenon in many developing countries, especially in China [3]. If a senior citizen suffers from an acute disease or injury, especially in outdoor environment, he/she must be treated immediately. Hence, it is essential to develop economical, convenient, and scalable communal healthcare systems, which can effectively reduce the response time of outdoor healthcare services and support the elderly community.

Providing an effective health system for senior citizens is significant, especially for those suffering from acute conditions. Therefore, increasing researchers focus on the topic and propose a great deal of valuable works. The research works in [4-8] focused on improving the healthcare systems using technological advances. The presented architectures for the communal healthcare systems are developed by introducing advanced digital technologies such as robot technology, cloud computing, wireless communication, and IoT, into the design framework $[9,10]$. These new technologies have contributed immensely to improving the existing healthcare system and providing effective health services [11, 12]. However, the existing healthcare frameworks are still not sufficient for providing an efficient service to the senior citizen community. The existing healthcare frameworks show slow first aid response time for the acute diseases or fall-related injuries of elderly due to the environment, non-user-friendly devices, traffic situations, and other obstacles. The three most important aspects of the senior citizen community healthcare system are as follows: (1) a fast response time for the first aid; it is known that if a senior citizen suffer from acute disease, the health system must respond within limited time; (2) an effective communication equipment and communication 
scheme; communication system is the basement for the new health framework, as effective communication equipment and scheme can promote the efficiency of whole system; (3) a set of user-friendly devices and user terminals. Lack of friendly terminal and devices is harmful for the efficiency and first aid response times. These new requirements bring new challenges for developing a healthcare system suitable for the elderly in outdoor environment, especially for response time and an effective communication system. Unfortunately, the traditional healthcare system cannot provide a fast access, efficient means, and valid communications system, which makes it difficult to meet the new requirements of the elderly healthcare system.

With the development of advanced technologies, such as smart robots, UAV, and wireless communication, it is possible to develop a new healthcare system to meet the demands of providing an efficient healthcare for elderly people in an outdoor environment. As discussed above, the existing healthcare systems are facing more challenges and cannot meet the requirements of providing highly efficient service and user-friendly devices and interface for the elderly community in outdoor environment. Hence, in this work, we propose a new outdoor healthcare system for senior citizens community by introducing the UAV and IoT. Moreover, we analyze the proposed healthcare architecture from the point of view of four layers: devices, communication, cloud, and terminal interface. Then, the proposed system is emulated in the terms of first aid response time and data communication delay in a simulation. A prototype is implemented using the designed healthcare UAV, user-friendly devices, and a related APP. Further, we build an experimental testbed and perform the experiment in a campus environment. The experimental results show that our proposed system can reduce the response time and provide a high-quality communications link. We believe that our work will shed light on outdoor healthcare system for senior citizens and accelerate the implementation of UAV and IoT-based healthcare services.

The rest of this paper is organized as follows. We present a summary of related works in Section 2. In Section 3, we introduce the system architecture and design of our proposed healthcare system for the elderly in outdoor environment and proposed an algorithm for health emergent event. We present the analysis of the proposed system and simulation and experimental results in Sections 4 and 5. Finally, conclusions of this work are given in Section 6.

\section{Related Work}

Combining advanced technologies to develop a smart healthcare system is an efficient method for providing an improved healthcare for the public, especially for senior citizens in modern society. These advanced technologies and systems enhance the performance of the healthcare services significantly. In this section, we review some of the related works of recent advances in emergency care technologies and healthcare systems.

2.1. Emergency Care Technologies. In [13], the authors integrated the RFID technology, ZigBee technology, and long-range wireless communication to build an emergency care system. Similarly, a global system for mobile (GSM) based smart wearable system with different sensors is developed in [14]. The developed device is capable of detecting sudden fall situations, cardiac abnormalities, and hyper/hypotension. In [15], Robson et al. proposed a tool called intelligent detection of arrhythmic heartbeats on electrocardiograms (IDAH-ECG). The IDAH-ECG tool collects data from an electrocardiograph to analyze and classify the data to detect patterns of arrhythmic beats in the ECG signal. In [16], the authors developed a location and emergency monitoring system for providing emergency care to the elderly. In [17], for predicting ambulance arrival time in an emergency situation, a linear regression model based on the information of weather, patient characteristics, lights and sirens, daylight, and rush-hour intervals is developed, and it was implemented as a Google maps web application.

From the perspective of application level, the novel technologies for emergency care could be divided into three categories: communication, data analysis, and data mining. However, the existing works related to emergency care technologies mainly focused on the healthcare service from a prediction aspect, which suffers from two undesirable drawbacks: real-time care deficiency and lack of emotional care. By incorporating UAV, IoT, and video technology, our proposed system can overcome these drawbacks.

2.2. Healthcare System. With the development of the information and communication technologies, such as wireless networks, cloud computing, data mining, and IoT, healthcare systems have made a great progress. In [18], a novel healthcare system architecture is proposed. The proposed architecture includes three components: collaborative data collection via wearable devices, enhanced sentiment analysis and forecasting models, and controllable affective interactions. In [18], a healthcare system based on cloud computing and robotics is developed, and it comprises wireless body area networks, a robot, a software system, and cloud platform. Further, in order to improve the practicability of multimedia transmission in the healthcare system, the authors in [19] proposed a novel scheme to deliver real-time video through an improved UDP-based protocol. In [20], a cyberphysical system for patient-centric healthcare applications and services, called Health-CPS, is proposed. The HealthCPS system is built based on cloud computing and big-data analytics technologies. In [21] a new system based on IoTaware smart architecture is developed for automatic monitoring and tracking of patients, personnel, and biomedical devices within hospitals and nursing institutes. In [22], the authors proposed a new healthcare system based on secondgeneration RFID systems characterized by the introduction of encoding rules that are dynamically stored in RFID tags.

From the described summary of the existing healthcare systems, it can be understood that various healthcare systems and devices are developed for accurately sensing and collecting data related to physical and mental health of users. However, there are several drawbacks for the existing healthcare systems. Firstly, the current healthcare system and the corresponding research mainly focus on a common 


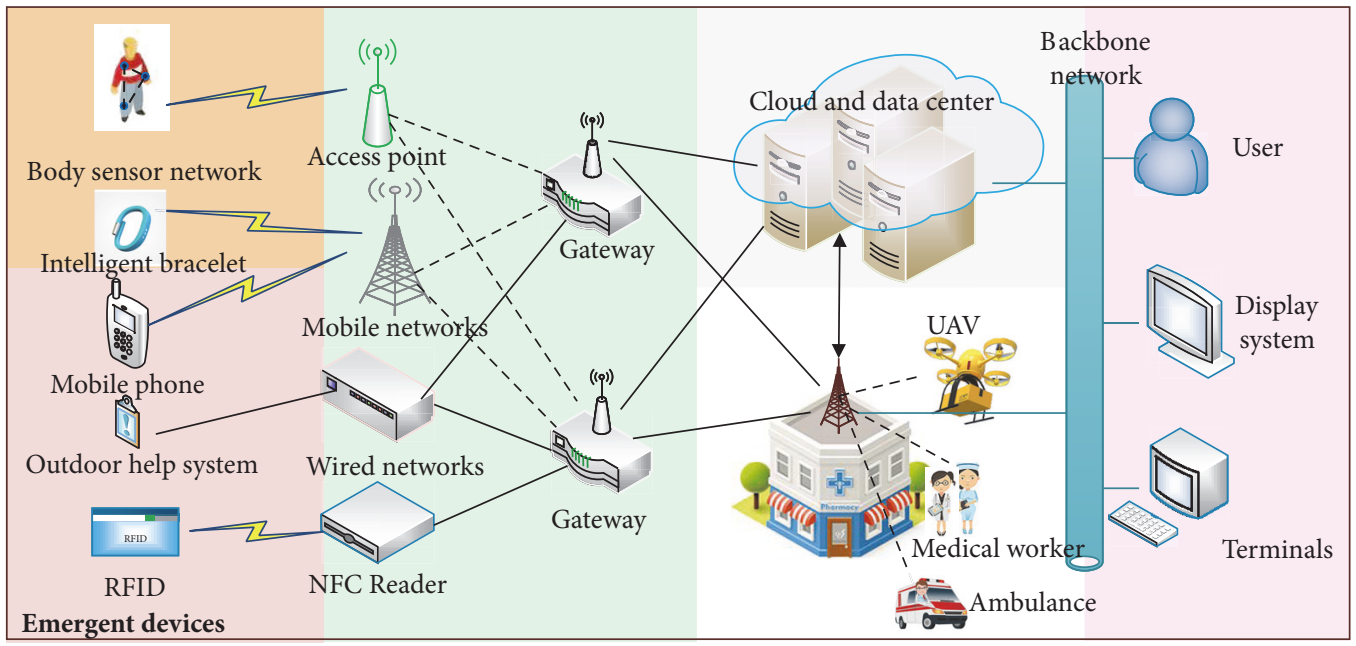

FIGURE 1: Architecture of network for senior citizen community in outdoor environment.

system for everyone and lack specific concrete applications, especially for senior citizen community outdoors. Moreover, the existing research studies assume that the users move in a limited range or at home. Obviously, this assumption is one of the limiting factors. In addition, for a healthcare system, it is important to have an efficient and real-time corresponding medical emergency subsystem, particularly for the users in emergency situations in outdoor environment. Nevertheless, the current emergency healthcare system is influenced by the position of hospital or medical center, buildings, and traffic situation. Hence, in this work, we focus on UAV and IoT- based emergency healthcare system for senior citizen community in outdoor environment.

\section{System Architecture}

In our work, UAV and IoT will play a significant role in the proposed healthcare system for outdoor environment. For facilitating the discussion and analysis of the framework, we divide the architecture of the outdoor healthcare system into four layers: the multiple model physical layer, networks layer, cloud layer, and the smart terminals (including UAV and medical workers) layer. The data communication links the four layers from the physical layer to the terminals. In the proposed system, the healthcare UAV plays a bridging role for information delivering during senior citizens and cloud sever; meanwhile UAV deliver the medicine, bandages, and emergency supplies. Therefore, in this section, after a brief description of the proposed healthcare system, the healthcare UAV subsystem is discussed.

3.1. Framework of the Proposed System. In the proposed system, all of the status data should be gathered by the sensor nodes and the outdoor system. Next, the data is transmitted to the cloud and data center. Meanwhile, the analyzed data can be sent to the smart terminals (e.g., doctors, users, smart phones, and other smart devices). It can be seen that the information exchange plays a significant role in our proposed healthcare system; i.e., the communication network supports the whole architecture. As shown in Figure 1, the information exchange system could be divided into data gathering layer, communication layer, cloud and data center, and smart terminals (including UAV and medical workers).

(1) Multimodel Data Gathering Layer. The data gathering layer determines the types of data that need to be collected by various devices in the system. The data gathering devices could be divided into two types, namely, forecasting and emergency devices. The body sensor nodes, the intelligent bracelet, environment sensors, and other sensors form the forecasting devices. The forecasting devices mainly gather the data for the general case and forecast the health condition of the users. The emergency devices are responsible for the emergency situations such as heart attack, hypertension, and other acute diseases. The emergency devices include the mobile phone, the outdoor help system node, RFID cards, and other smart devices.

(2) Communication Layer. This layer is responsible for communication between the cloud and smart devices, hospital system, and the users. The communication layer uploads and delivers the sensing data, control commands, and other information. In the proposed system, the communication media platform uses both the wired and wireless communication technologies. The wired network mainly deals with the static nodes such as outdoor help system, real-time data transfer, and situations with large amounts of data transfer. For the mobile entities (such as the ambulance, UAV, medical workers, and users), wireless communication technologies such as Bluetooth, ZigBee, Wi-Fi, LTE, and NFC are used in the system.

(3) Cloud and Data Center. The cloud and data center perform computing to match the users' requirements and store massive amount of information. Moreover, the cloud and data center act as the processing module for all the networks. In an emergency situation, the cloud and data 
center create the list of the key medicines and rescue devices, by comparing the current information and the stored medical history records of a patient. The Hadoop software is used for storing massive amount of user data. After data mining and analysis, the results are delivered to users, nearby hospital, medical workers, and other smart terminals based on the priority of the message.

(4) Smart Terminals. The smart terminals are used to display the related information and key data using messages, web pages, or other application. This interface could provide an intuitional, effective, personal, and visual result for the elderly community and medical workers. The smart terminals can be the mobile phone, the intelligent bracelet, and the display system. For example, with the application software installed on the smart phone, the user could check the monitoring status of health conditions such as heart-rate, blood pressure, and environment data. When real-time information is issued from data server, the reminders will be driven by sound and video.

3.2. Problem Formulation and First Aid Strategies Based on $U A V$. In our system, two types of data traffic are considered: (1) general data, where all of the data are delivered in normal situation; namely, the elders are not in emergency; (2), emergent data, where the data are delivered in emergent situation, that is, the elders being in a critical situation.

Assume that there are $n$ senior citizens randomly distributed in $A(L, W)$, where $\mathrm{L}$ and $\mathrm{W}$ are are length and width of $A$. Let $\left(x_{i}, y_{i}\right)$ be the position of the $i$-th senior citizen. For the system model, given that a senior citizen (we use current node $i$ which stands for the senior citizen) is falling into the emergent situation at the time $t_{0}$ and the first aid at time $t_{\text {arrived }}$. So, the first aid response time $T$ is

$$
T=t_{\text {arrived }}-t_{0}
$$

Let the coordinate of medical center be $\left(x_{0}, y_{0}\right)$ and $\left(x_{e}, y_{e}\right)$ be the position of emergent event. Let $l$ denote the length of path for ambulance from $\left(x_{0}, y_{0}\right)$ to $\left(x_{e}, y_{e}\right)$. So, the direct length of medical center and emergent event site $l_{\mathrm{d}}$ can be described as follows:

$$
l_{d}=\sqrt{\left(x_{0}-x_{e}\right)^{2}+\left(y_{0}-y_{e}\right)^{2}} .
$$

To better understand the solution to a problem, establish the relationship between $l$ (real path length) and $l_{d}$, shown in the following:

$$
l=(1+\gamma) l_{d}
$$

where $\gamma$ is path length increase coefficient and the value of $\gamma$ depends on different method for the emergent situation. So, the first aid response time $T$ is formulated as follows:

$$
T=t_{\text {recieve_RQ }}+t_{\text {arrived_AM }}+t_{\text {data_process }}
$$

where $t_{\text {recieve } R Q}$ is the communication delay for the emergency request information (ERI) arriving to medical center. $t_{\text {arrived_AM }}$ denotes the time of the ambulance arriving at the emergent site; $t_{\text {data_process }}$ is the data processing for dealing with the healthcare emergent event. Moreover, in the communication network, $t_{\text {recieve_RQ }}$ can be descripted as

$$
t_{\text {recieve_RQ }}=\sum_{j=1}^{k}\left(\frac{Q}{V_{C, j}}+t_{\text {wait }, j}\right)
$$

where $Q$ is the data size of ERI and $V_{c, j}$ and $t_{\text {wait }, j}$ are communication rate and unload communication task time of the $j$-th relay node. For easy understanding, let $V_{c, j}$ and $t_{\text {wait }, j}$ have the same value; namely, $V_{c, j}=V_{c}, t_{\text {wait }, j}=t_{\text {wait }}$. So, (5) can be reduced to

$$
t_{\text {recieve_RQ }}=k\left(\frac{Q}{V_{C}}+t_{\text {wait }}\right)
$$

Let $V_{m}$ be the speed of ambulance. It is known that there is a relationship between real path length $(l)$ and ambulance moving speed $\left(V_{m}\right)$, as shown in the following:

$$
t_{\text {arrived_AM }}=\frac{l}{V_{m}} .
$$

So, formulation (4) can simplify into

$$
T=k\left(\frac{Q}{V_{C}}+t_{\text {wait }}\right)+\frac{l}{V_{m}} .
$$

As discussed above, in the emergent situation, we hope to get the minimum response time (MRT). So, the problem of MRT can be formulated as (9):

$$
\begin{array}{ll}
\min & T \\
\text { s.t. } & l \geq l_{d} \\
& V_{m} \leq V_{m, \max } \\
& 0 \leq \gamma \leq \gamma_{\max } \\
& \mathrm{Q}>0 \\
& \left(x_{0}, y_{0}\right),\left(x_{e}, y_{e}\right) \in A \\
& t_{\text {wait }}>0, V_{c}>0
\end{array}
$$

where formulations (10), (11), (12), (13), (14), and (15) are the constraints of length of medical center and emergent event site, and ambulance moving speed, path length increase coefficient, data size of ERI, position of emergent event, communication rate, and unload communication task time, respectively.

On the grounds of the processing of dealing with the healthcare emergent event and the above discussion, problem (9) can be decoupled into the following three subproblems, as being formulation as

$$
\min T=\min \left(t_{\text {recieve_RQ }}+t_{\text {arrived_AM }}+t_{\text {data_process }}\right)
$$

According to formulations (6) and (7), the optimal problem (9) can be further simplified into (17)

$$
\begin{aligned}
\min T= & \min (k)\left(\frac{\mathrm{Q}}{V_{C}}+t_{\text {wait }}\right)+\frac{\min (l)}{V_{m}} \\
& +\min \left(t_{\text {data_process }}\right)
\end{aligned}
$$




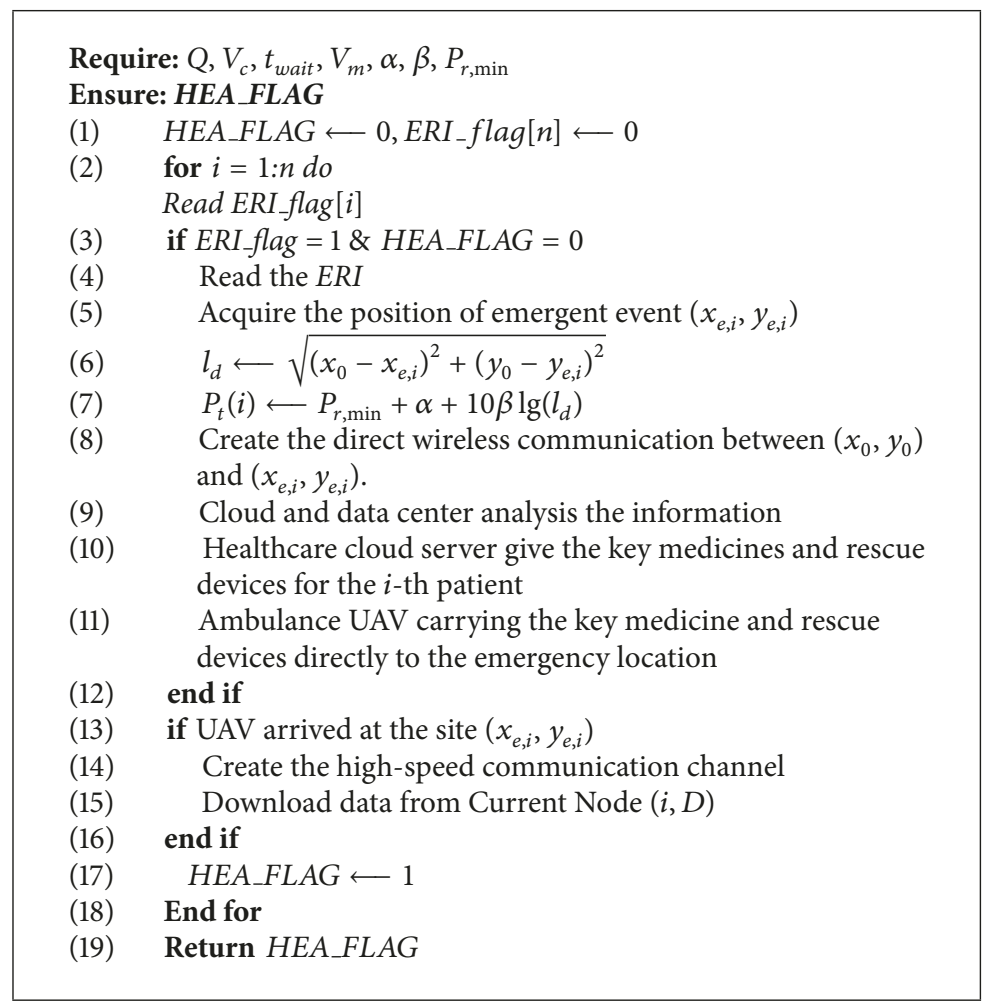

Algorithm 1: First aid in emergent situation based on UAV.

In other words, for getting the first aid response time $T$, we can reduce the relay number of $k$ and decrease the real path length $l$. Furthermore, in wireless mobile network, the receiving power $P_{r}$ must satisfy the following inequality:

$$
P_{r}=P_{t}-\alpha-10 \beta \lg (R) \geq P_{r, \text { min }}
$$

where $P_{t}$ is transmitting power; $R$ is the wireless communication radius; $\alpha$ and $\beta$ are the attenuation at reference distance and the path loss exponent, respectively; $P_{r, \min }$ is the minimum receiving power for wireless communication. For solving the first subproblem $(\min (k))$, we can create the direct wireless communication between $\left(x_{0}, y_{0}\right)$ and $\left(x_{e}, y_{e}\right)$.

So, for acquiring the minimum data exchanging time, we can turn the first subproblem into searching the minimum transmitting power.

$$
\min P_{t}=P_{r, \min }+\alpha+10 \beta \lg \left(l_{d}\right)
$$

According to the above analysis, we propose a novel strategy for dealing with the health emergent event. For dealing with problem (9) with constraints of (10)-(15), we proposed the new healthcare system by using UAV, IoT, and cloud server. The strategy is shown in Algorithm 1.

Our proposed algorithm is explained as follows. When a senior citizen is in an emergency condition, he/she and the finder could send the request (ERI_flag $[i])$ for help by using the outdoor help network terminal, intelligent bracelet, and other smart terminals (shown using the red line in
Figure 2). Once the network receives the emergency request information, it creates a real-time wireless channel to deliver the information. By comparing the current information and the recorded history, the cloud and data center can give information regarding list of the key medicines and rescue devices for the patient. The hospital data center drives the ambulance UAV carrying the key medicine and rescue devices directly to the emergency location $\left(x_{e, i}, y_{e, i}\right)$. Meanwhile, the cloud and data center inform the nearest medical workers and doctors requesting assistance at the site. When the UAV arrives at the emergency location, the wireless link will establish a connection with the hospital center, medical workers, and doctors. Meanwhile, the related emotional music will play, and the video phone will be linked, according the specific situation. Then, the finder and the nearest medical worker could give a medical care.

As shown in Algorithm 1, when UAV arrived at site, UAV will create the high-speed wireless channel for delivering video or other key data. Our proposal can reduce the data processing time by adopting cloud server f. Let $V_{c}$ be the wireless communication rate and $D$ be the volume of delivering data. So, we can get the communication delay, as shown in

$$
T_{\text {communication_delay }}=\theta \frac{D}{V_{c}}
$$

where $\theta$ is the average number of hops for delivering data $D$. It is easily known that in our proposal we use UAV to create two hops for finishing the data transmitting. 


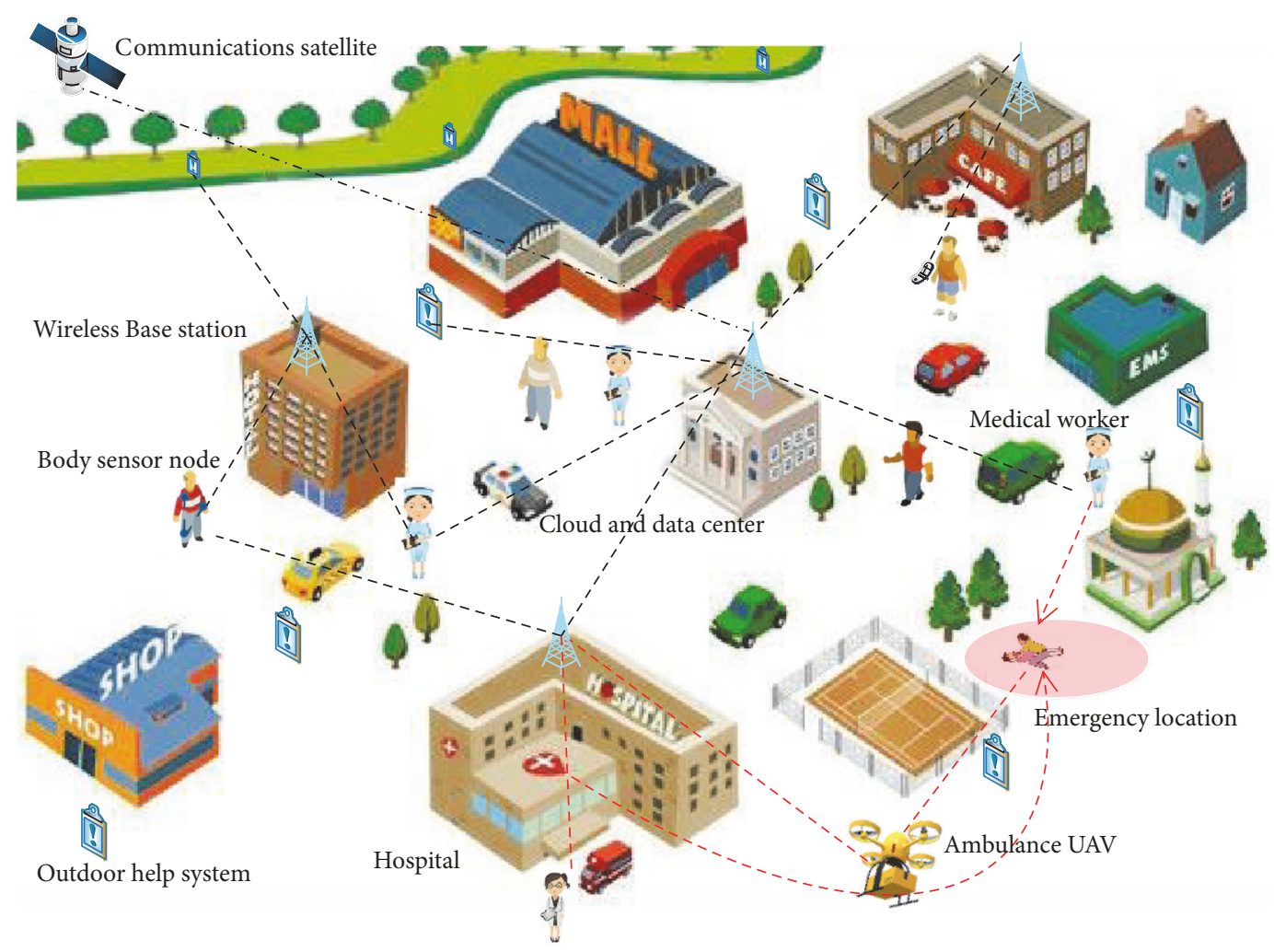

FIGURE 2: The outdoor healthcare system for elderly community. The red line shows the emergency situation case.

TABLE 1: Simulation parameters.

\begin{tabular}{lccc}
\hline Parameters & values & Parameters & values \\
\hline$(x, y)$ & $(0,0)$ & $\alpha$ & -30 \\
$\gamma_{H S U A V}$ & 0 & $t_{\text {data_process }}(R T M E)$ & 2 \\
$t_{\text {data_process }}(H S U A V)$ & $10 \mathrm{~s}$ & $\theta_{R T M E}, \theta_{S M H S}$ & $10 \mathrm{~s}$ \\
$t_{\text {data_process }}(S M H S)$ & $100 \mathrm{~s}$ & $P_{r, \text { min }}$ & 2,4 \\
$Q$ & $1 \mathrm{Mb}$ & $r_{T M H C}$ & $-100 \mathrm{dBm}$ \\
$t_{\text {wait }}$ & $1 \mathrm{~ms}$ & $l_{d}$ & $1 \sim 2$ \\
$\theta_{\text {HSUAV }}$ & 1 & $V m$ & $100 \sim 1000 \mathrm{~m}$ \\
$V c$ & $1 \mathrm{Mb} / \mathrm{s}$ & $V^{2}$ & $2 \mathrm{~m} / \mathrm{s}$ \\
$D$ & $100 \mathrm{Mb} \sim 1000 \mathrm{Mb}$ & $50 \mathrm{~m}$ \\
\hline
\end{tabular}

\section{Performance Evaluation}

In this section, we will evaluate the performance of the proposed healthcare architecture and algorithm by comparing with traditional method in terms of first aid response time and communication delay.

The strategies are simulated in related software. Without loss of generality, we assume that the healthcare center is at the origin of coordinate system (namely, $(x, y)=(0,0)$ ). The distance between emergent healthcare event position and origin of coordinate system will increase for evaluating the performance index. For easy reading, we use HSUAV which stands for our proposed method and RTME and SMHS which stand for the healthcare method in [23] and [24], respectively. In the simulation setup, let speed of the ambulance vehicle of HSUAV, RTME, and SMHS be $2 \mathrm{~m} / \mathrm{s}$. The details of the remaining parameters are listed in Table 1.

The simulation results of first aid response time and communication delay in different healthcare frameworks are given in Figure 3. The first aid response time total time consumption is the best standard metric for different architecture. It is known that, with increase of $l_{d}$, the first aid response time $T$ of the three strategies will be added, as shown in Figure 3(a). However, HSUAV will use less time than RTME and SMHS. When $l_{d}=1000 \mathrm{~m}, \mathrm{HSUAV}$ can reduce by $30 \%$ and $48 \%$ first aid response time comparing with RTME and SMHS. In other words, in our proposal framework, we can get a higher efficiency for healthcare emergent event.

Similar performance results of communication delay can be observed in Figure 3(b). In general, communication delay 


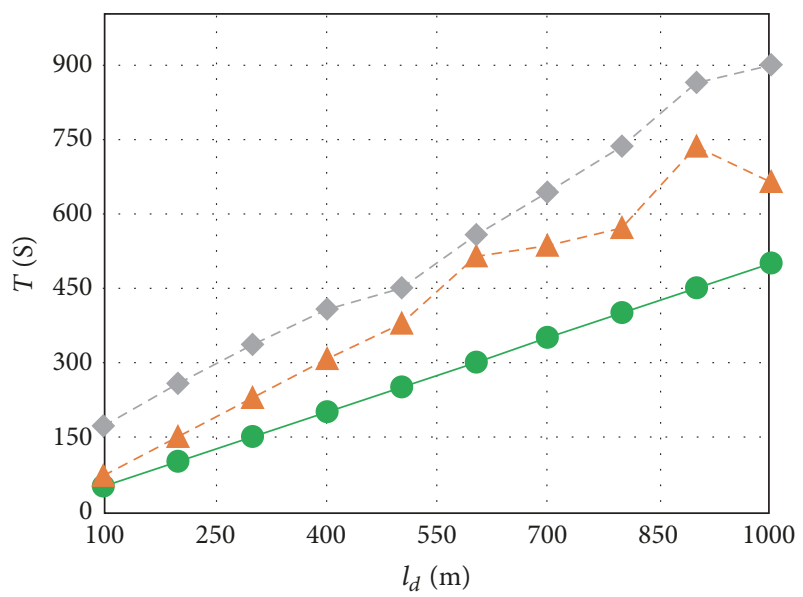

$$
\begin{aligned}
& \text { HSUAV } \\
& \text { - RTME } \\
& \text { SMHS }
\end{aligned}
$$

(a) First aid response times

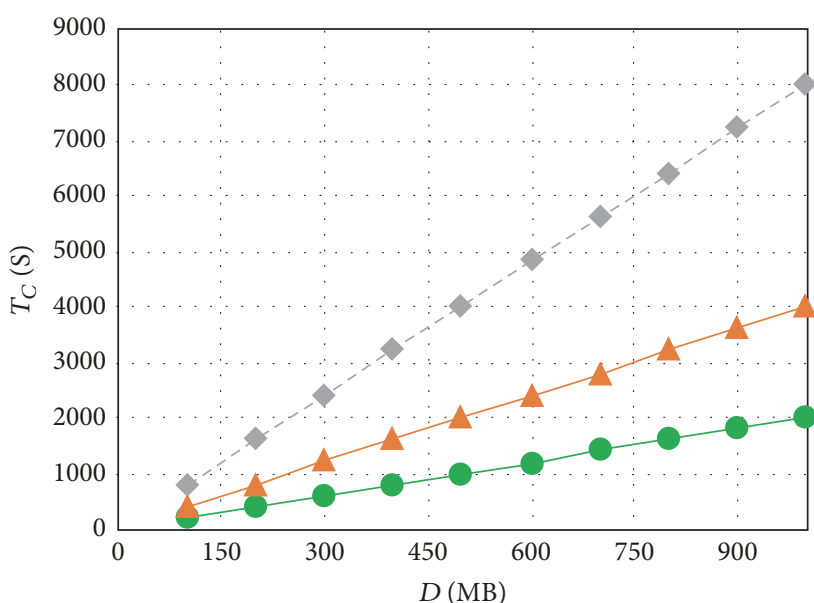

- HSUAV

RTME

SMHS

(b) Communication delay in different loads

FIGURE 3: First aid response times and communication delays in different ways.

of HSUAV, RTME, and SMHS will increase with the rising of transmission data volume. The results in Figure 3(b) indicate that HSUAV has the smaller average time consumption for data delivering. In short, our proposed method outperforms the traditional framework in terms of first aid response time and communication delay.

\section{Experiment Testbed}

5.1. Healthcare UAV Subsystem. The ambulance UAV is used in the platform for reducing the response time. The UAV could directly fly to the emergency site, without being affected by the traffic situation. In order to implement the UAV system, dual-microprogrammed control units (MCUs) are used, as shown in Figure 4. Using PWM (Pulse Width Modulating, PWM), the MCU controls the motor, and propellers. At the same time, air velocity (MS-5611), working current, and voltage could be monitored by corresponding sensors. Further, using the universal asynchronous receiver/transmitter (UART) and serial peripheral interface (SPI), the UAV MCU configures and controls the GPS and wireless communication module for remote controlling (433 MHz DTU).

The sub-MCU connects the UAV MCU using the I2C interface. The sub-MCU accesses the loudspeaker, camera, SD card, ambient light, and environment sensors for the corresponding information. When the UAV flies to the emergency site, the main MCU sends a signal from the site and then the sub-MCU begins to work. At first, the subMCU establishes the wireless communication links with the hospital data center and the doctor. Next, some emotional music and conversations will be sent by the program processed in the sub-MCU. Further, the sensing data, images, videos, voice, and other key parameters will be sent to the data center and other related devices.
On the one hand, the sub-MCU could receive the emergency commands from the wireless module. When an emergency command is received by the hospital data sever, after being equipped with corresponding medicine and based on the patient information, the sub-MCU sends the GPS position data to the main UAV MCU. The main UAV MCU will fly to the emergency site. On the other hand, when the UAV arrives at the site, it could transmit the real-time video to hospital, ambulance, and medical workers. Meanwhile, the UAV can play music and sound to alleviate psychological pressure. In some special situations, the doctor could give assisted help by the remote video communication from the hospital. It is important to note that the wireless communication link can be supported by outdoor help system network, $\mathrm{Wi}-\mathrm{Fi}$, or LTE.

5.2. Implementation of Prototype. In this section, we briefly introduce an effective UAV and IoT-based healthcare service prototype developed for testing in a smart campus use case scenario as shown in Figure 5. This prototype system includes serval smart devices, such as cloud data center, wireless nodes, and display screen. Meanwhile, a four-axis UAV is used as the ambulance UAV. The UAV is equipped with different sensor nodes and devices, such as GPS, wireless modules, and different kinds of sensors. The main UAV MCU is equipped with an 8-bit ATMEGA2560-16AU microcontroller unit with AVR architecture, a 256 KB RAM memory, and a CPU with $16 \mathrm{MHz}$ speed. The sub-MCU board is used for supporting the functions described above. In our ambulance UAV, for reducing the energy consumption, a 32-bit ST STM32F427VIT6 microcontroller unit with ARM Cortex-M4 architecture, a $2048 \mathrm{~KB}$ of flash memory, a 256 KB RAM memory, and a CPU with $168 \mathrm{MHz}$ speed are used. Wireless communication capabilities are provided by the IEEE 802.15.4 TI (CC2530) chip with the working frequency 


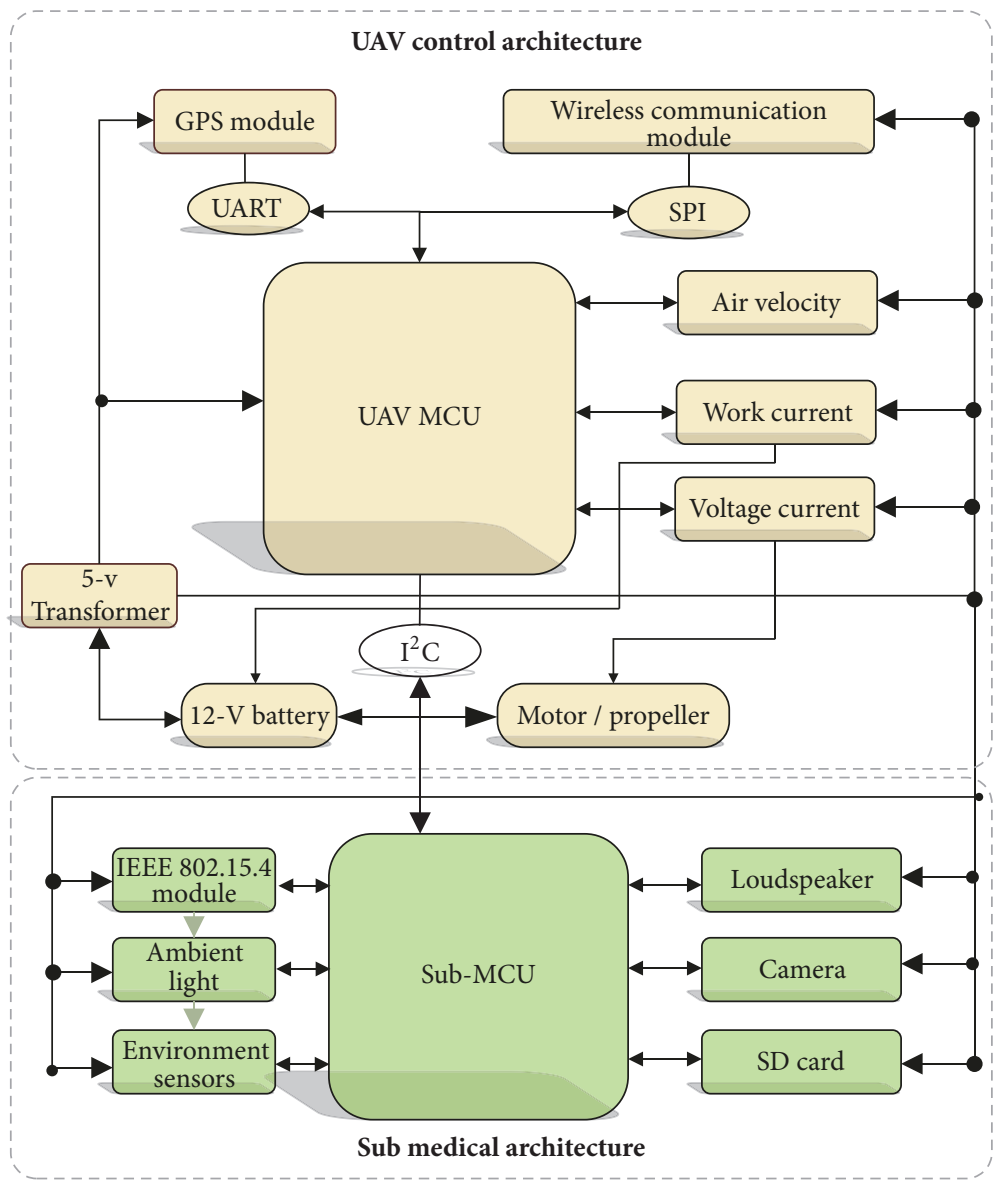

FIgURE 4: The UAV system hardware architecture.

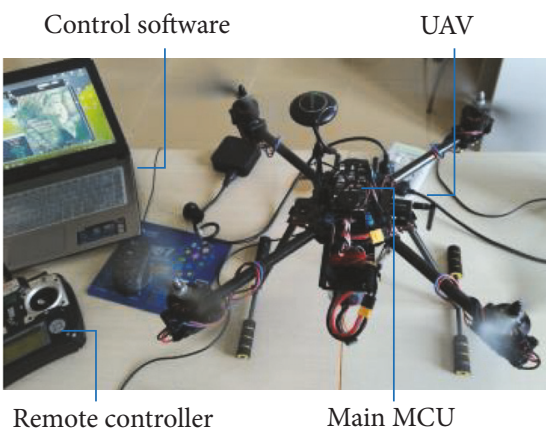

(a) Ambulance UAV

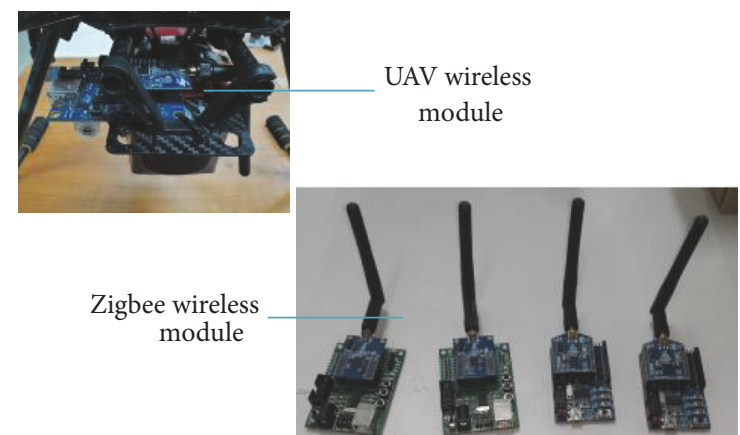

(b) The prototype system

FIGURE 5: The platform and ambulance UAV.

of $2.4 \mathrm{GHz}$. Hence, a simple ZigBee network is established on the campus.

The smart terminal software is developed based on the android development kit with the android operating system with version 4.5 . The emergency situation information and the collected sensory data can be displayed on smart phone by the corresponding application. Figure 6 shows the environmental data and body physiological data captured by various sensors in a visualization chart. Figure 6 shows a set of screenshots of the medical application used on the phone, the application has the option to display GPS position, emergency message, heart-rate, and blood pressure information. Figure 6(a) shows main page of the user terminal, and it includes various parameters such body temperature, heartrate, location, and environmental data. The histogram of averaging humidity per minute is also displayed as shown in Figure 6(b). Furthermore, the body temperature illustration is shown in Figure 6(c). 


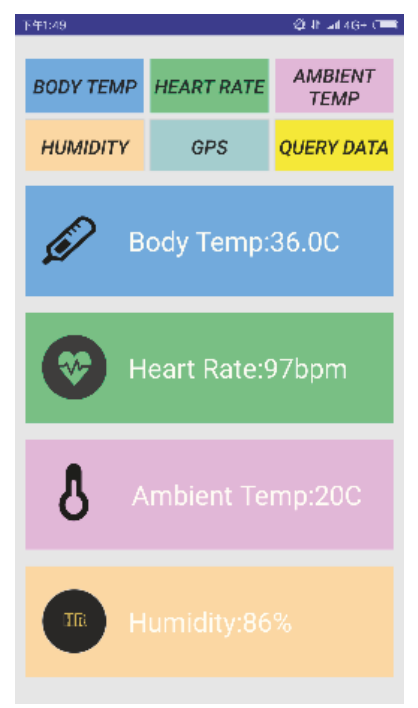

(a)

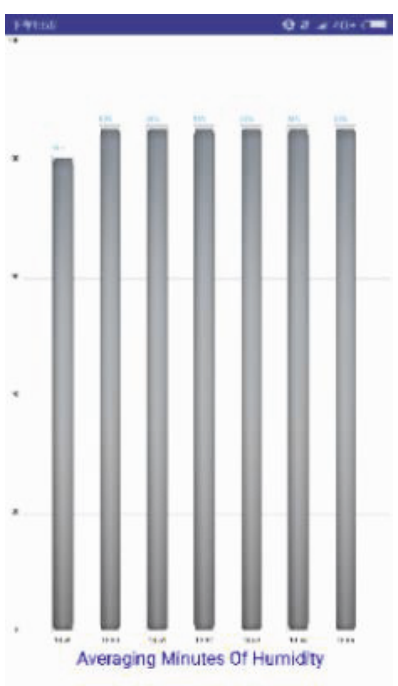

(b)

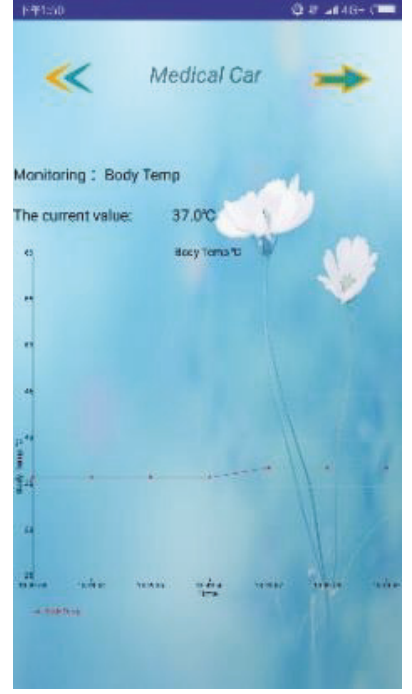

(c)

FIGURE 6: Screenshots of the medical app on the smart phone.

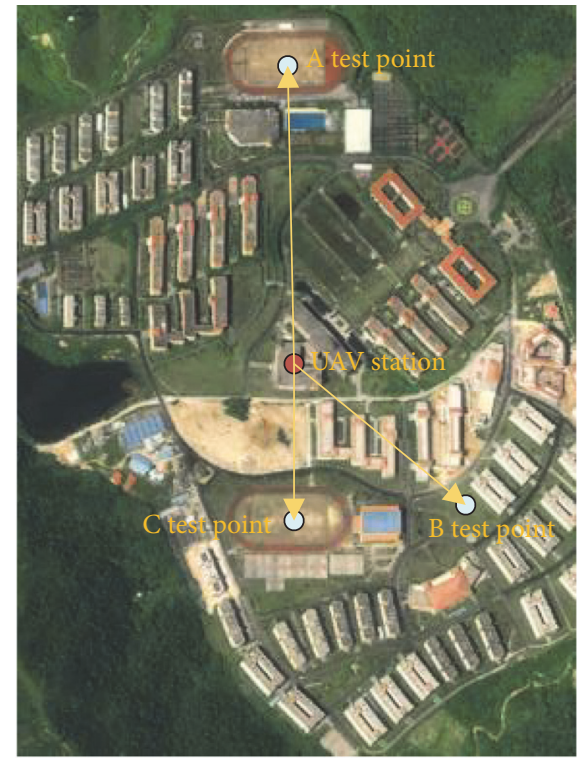

(a) The diagram of experiment location

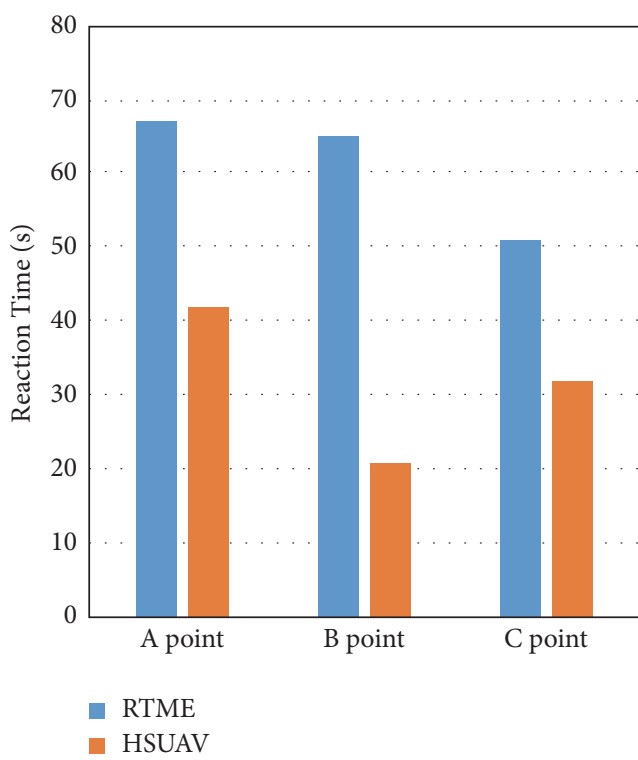

(b) The result of RT for different schemes

Figure 7: Comparison of reaction time for different systems.

\subsection{Experiment Results and Analysis}

First Aid Reaction Time. For assessing the reaction time performance of our proposed healthcare system, a comparison between the proposed system and other traditional architectures is carried out in our campus. We assumed that the UAV and the traditional medical ambulance have the same speed of $36 \mathrm{Km} / \mathrm{h}$. We choose three different distance points as the emergency sites as shown in Figure 7(a). Figure 7(b) illustrates the comparison result. From the figure, our proposed system can reduce the response time by $20 \%$. Our proposed system has a fast reaction time compared to
RTME. Results show that the proposed system outperforms the traditional architectures, which can be easily affected by building, traffic, and road network. However, the UAV could fly directly to destination without any obstacles.

\section{Conclusion}

In this paper, we considered the outdoor healthcare system for senior citizens in emergency situations. For reducing the response time in case of an emergency situation, a healthcare system based on UAV and IoT is designed. The proposed system can provide real-time help to the senior citizens in 
outdoor emergency situations with the help of the UAV. Moreover, it can also deliver the emergency information using the communication networks and assist specialist or medical workers. The proposed system includes four functions: sensing, communication, data processing, and medical aid. Since we used a UAV in the system, the proposed system is a closed loop system. Moreover, we discussed the communication architecture of the proposed system from the perspective different layers. Finally, a testbed for the outdoor healthcare system has been presented and functional validation and performance validation are presented. The experimental results show that the proposed system outperforms the traditional emergency care system architectures. Simulation and experimental results show that the proposed system outperforms the current architectures, which can be easily affected by building, traffic and road network. However, the UAV could fly directly to destination without any obstacles.

\section{Data Availability}

The simulation results data and experimental data can be accessed from the website, https://pan.baidu.com/s/ 1HVw1xZ2VLrpoLW6W8tuskw, or acquired from the corresponding author upon request.

\section{Ethical Approval}

All procedures performed in studies involving human participants were in accordance with the ethical standards of the institutional and/or national research committee and with the 1964 Helsinki declaration and its later amendments or comparable ethical standards.

\section{Consent}

Informed consent was obtained from all individual participants included in the study.

\section{Conflicts of Interest}

The authors declare that they have no conflicts of interest.

\section{Acknowledgments}

This work is supported by the Youth Innovation Project of Important Program for College of Guangdong Province with no. 2015KQNCX228 and Guangzhou Science and Technology Program (no. 201804010427). This work was also partly supported by the colleagues in the Department of Electronic Communication \& Software Engineering Nanfang College of Sun Yat-sen University and School of Information Science and Technology, South China Agricultural University.

\section{References}

[1] W. C. Mann, “The aging population and its needs," IEEE Pervasive Computing, vol. 3, no. 2, pp. 12-14, 2004.
[2] H. M. Holmes, M. S. Beck, and J. H. Rowe, "Geriatrics: Year in review," Journal of Geriatric Oncology, vol. 7, no. 5, pp. 404-408, 2016.

[3] Y. Li, S. Wang, J. Li et al., "A survey of physicians who care for older persons in Southwest China," Journal of Nutrition Health Aging, vol. 17, no. 2, pp. 192-195, 2013.

[4] P. A. Laplante, M. Kassab, N. L. Laplante, and J. M. Voas, "Building Caring Healthcare Systems in the Internet of Things," IEEE Systems Journal, pp. 1-8, 2017.

[5] L. Wan, G. Han, L. Shu, and N. Feng, "The critical patients localization algorithm using sparse representation for mixed signals in emergency healthcare system," IEEE Systems Journal, vol. 12, no. 1, pp. 52-63, 2015.

[6] B. P. Zeigler, "Discrete Event System Specification Framework for Self-Improving Healthcare Service Systems," IEEE Systems Journal, vol. 12, no. 1, pp. 196-207, 2018.

[7] Y. Ma, Y. Wang, J. Yang, Y. Miao, and W. Li, "Big Health Application System based on Health Internet of Things and Big Data," IEEE Access, vol. 5, pp. 7885-7897, 2017.

[8] A. Srivastava, N. Sankar K., B. Chatterjee et al., "Bio-WiTel: A Low-Power Integrated Wireless Telemetry System for Healthcare Applications in 401-406 MHz Band of MedRadio Spectrum," IEEE Journal of Biomedical and Health Informatics, vol. 22, no. 2, pp. 483-494, 2018.

[9] M. M. Baig, H. GholamHosseini, and M. J. Connolly, "Mobile healthcare applications: system design review, critical issues and challenges," Australasian Physical \& Engineering Sciences in Medicine, vol. 38, no. 1, pp. 23-38, 2015.

[10] J. Wan, C. Zou, S. Ullah, C.-F. Lai, M. Zhou, and X. Wang, "Cloud-Enabled wireless body area networks for pervasive healthcare," IEEE Network, vol. 27, no. 5, pp. 56-61, 2013.

[11] H. Qi, M. Stojmenovic, K. Li, Z. Li, and W. Qu, "A low transmission overhead framework of mobile visual search based on vocabulary decomposition," IEEE Transactions on Multimedia, vol. 16, no. 7, pp. 1963-1972, 2014.

[12] Y. Sang, H. Qi, K. Li, Y. Jin, D. Yan, and S. Gao, "An effective discretization method for disposing high-dimensional data," Information Sciences, vol. 270, pp. 73-91, 2014.

[13] H. Shieh, C. Huang, F. Lyu, Z. Zhang, and T. Zheng, "An Emergency Care System Using RFID and Zigbee Techniques," in Proceedings of the 2016 International Symposium on Computer, Consumer and Control (IS3C), pp. 65-68, Xian, China, July 2016.

[14] S. Tewary, S. Chakraborty, J. Majumdar et al., "A novel approach towards designing a wearable Smart Health Monitoring System measuring the vital parameters and emergency situations in real-time and providing the necessary medical care through telemedicine," in Proceedings of the IEEE Students' Conference on Electrical, Electronics and Computer Science, SCEECS 2016, pp. 1-8, Bhopal, India, March 2016.

[15] R. Pequeno, N. Carvalho, K. Galdino et al., "A Portable System to Support Electrocardiography in Emergency Care," in Proceedings of the 2015 IEEE 28th International Symposium on Computer-Based Medical Systems (CBMS), pp. 256-257, Sao Carlos, Brazil, June 2015.

[16] C. Park and J. Kim, "A location and emergency monitoring system for elder care using ZigBee," in Proceedings of the 2011 7th International Conference on Mobile Ad-hoc and Sensor Networks, MSN 2011, pp. 367-369, China, December 2011.

[17] R. J. Fleischman, M. Lundquist, J. Jui, C. D. Newgard, and C. Warden, "Predicting ambulance time of arrival to the 
emergency department using global positioning system and Google maps," Prehospital Emergency Care, vol. 17, no. 4, pp. 458-465, 2013.

[18] M. Chen, Y. Zhang, Y. Li, M. M. Hassan, and A. Alamri, "AIWAC: affective interaction through wearable computing and cloud technology," IEEE Wireless Communications Magazine, vol. 22, no. 1, pp. 20-27, 2015.

[19] Y. Ma, Y. Zhang, J. Wan, D. Zhang, and N. Pan, "Robot and cloud-assisted multi-modal healthcare system," Cluster Computing, vol. 18, no. 3, pp. 1295-1306, 2015.

[20] Y. Zhang, M. Qiu, C.-W. Tsai, M. M. Hassan, and A. Alamri, "Health-CPS: Healthcare cyber-physical system assisted by cloud and big data," IEEE Systems Journal, vol. 11, no. 1, pp. 8895, 2015.

[21] L. Catarinucci, D. de Donno, L. Mainetti et al., "An IoT-aware architecture for smart healthcare systems," IEEE Internet of Things Journal, vol. 2, no. 6, pp. 515-526, 2015.

[22] M. Chen, S. Gonzalez, V. Leung, Q. Zhang, and M. Li, "A 2GRFID-based e-healthcare system," IEEE Wireless Communications Magazine, vol. 17, no. 1, pp. 37-43, 2010.

[23] M. M. Rathore, A. Ahmad, A. Paul, J. Wan, and D. Zhang, "Realtime Medical Emergency Response System: Exploiting IoT and Big Data for Public Health," Journal of Medical Systems, vol. 40, no. 12, article no. 283, 2016.

[24] Y. Ren, R. Werner, N. Pazzi, and A. Boukerche, "Monitoring patients via a secure and mobile healthcare system," IEEE Wireless Communications Magazine, vol. 17, no. 1, pp. 59-65, 2010. 


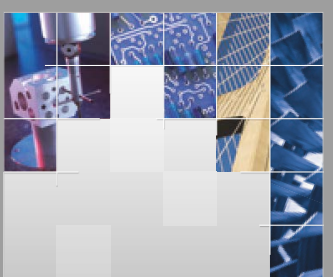

\section{Enfincering}
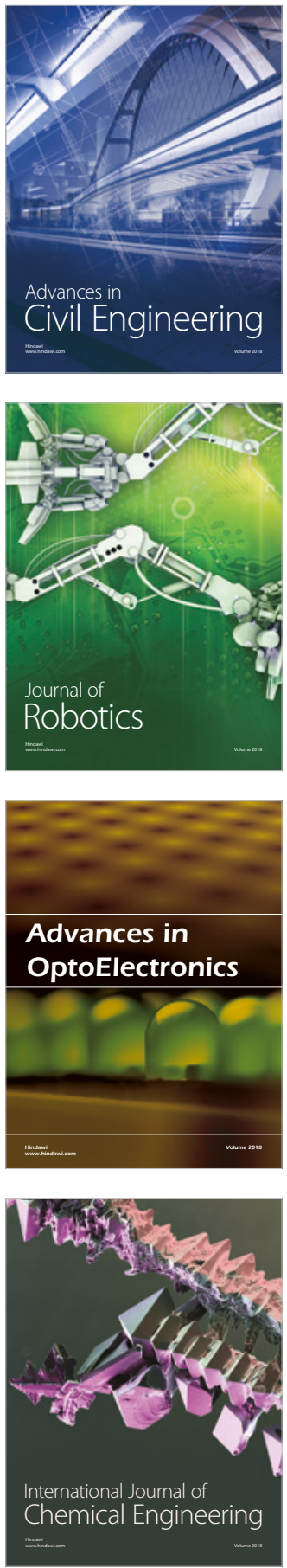

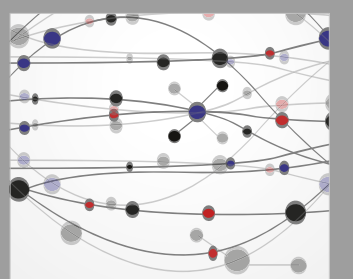

\section{Rotating \\ Machinery}

The Scientific World Journal

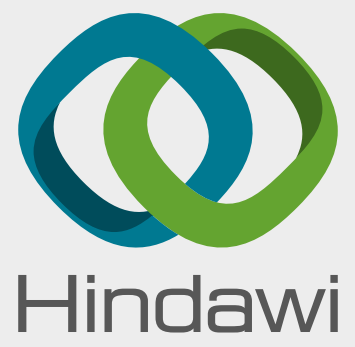

Submit your manuscripts at

www.hindawi.com
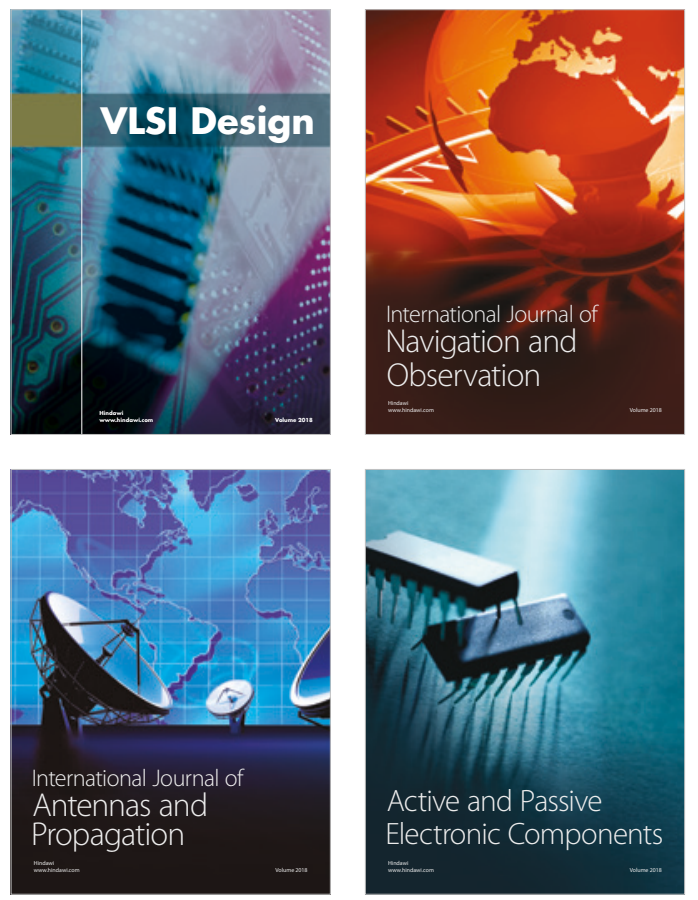
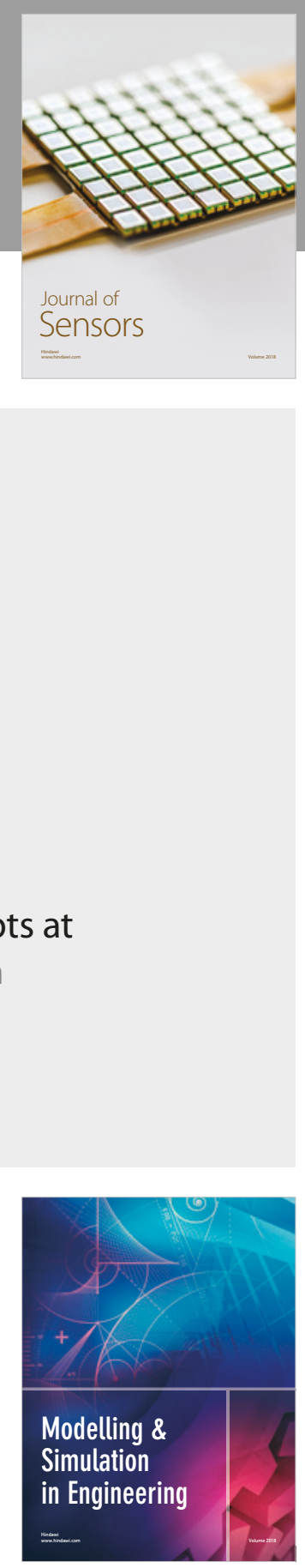

\section{Advances \\ Multimedia}
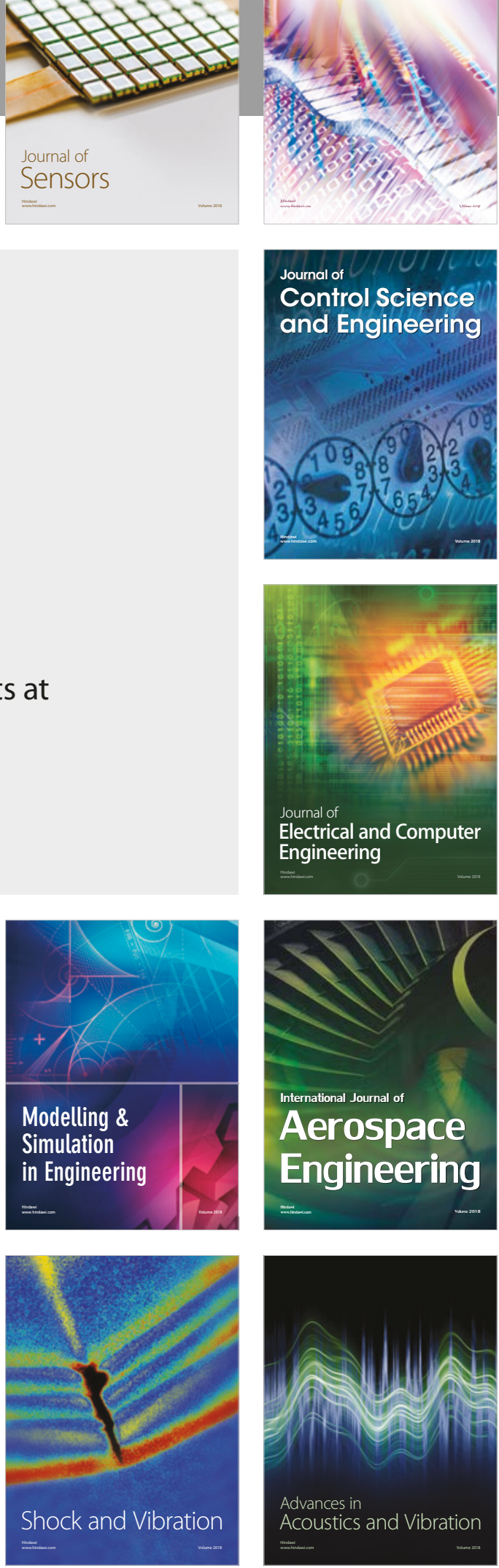